\title{
JUSTIÇA RESTAURATIVA: COVID-19 E SAÚDE MENTAL
}

\section{RESTORATION JUSTICE: COVID-19 AND MENTAL HEALTH}

\author{
Renata Luciane Polsaque Young Blood* \\ Nei Alberto Salles Filho** \\ Marcelo Rezende Young Blood***
}

\section{RESUMO}

Este artigo apresenta a experiência do Núcleo de Prática Jurídica do Centro Universitário Santa Amélia, em Ponta Grossa-PR, na aplicação de círculos virtuais de construção de paz no contexto do isolamento social provocado pela pandemia do COVID-19. O círculo é um método da Justiça Restaurativa, aqui definido como um programa simples, mas profundo, para construir relacionamentos mais significativos e ampliar a consciência emocional. Os impactos psicológicos negativos da quarentena motivaram a migração dos círculos presenciais para online, entendendo que a prática circular pode contribuir para a construção de rede de apoio e no fortalecimento de vínculo entre as pessoas. Com este objetivo foram realizados, em três semestres, 96 círculos, envolvendo 738 pessoas, e o resultado foi a percepção de que o círculo, mesmo virtualmente, é capaz de promover o autoconhecimento e a conexão interpessoal de uma forma profunda e significativa, contribuindo positivamente com a saúde mental na pandemia.

Palavras-chave: Círculo de Construção de Paz; Visão Holística; Virtual.

\footnotetext{
*Bacharel em Direito pela Universidade Federal do Paraná (UFPR). Especialista em Gestão Empresarial pelo Instituto Superior de Administração e Economia e Mercosul/Fundação Getúlio Vargas (ISAE/FGV). Mestre em Ciências Sociais Aplicadas pela Universidade Estadual em Ponta Grossa (UEPG). Doutoranda em Ciências Sociais Aplicadas pela Universidade Estadual de Ponta Grossa (UEPG). Facilitadora e Instrutora da Metodologia da Justiça Restaurativa pelo Tribunal de Justiça do Estado do Paraná. Mediadora pelo Tribunal de Justiça do Estado do Paraná. Coordenadora do Núcleo de Prática Jurídica da UNISECAL. Coordenadora de Pesquisa em Justiça Restaurativa do Curso de Direito da UNISECAL. Integrante do Grupo de Pesquisa intitulada: Direitos Humanos, Desenvolvimento Sustentável e Cultura de Paz: análise dos documentos de Organismos Internacionais à luz da Ciências Sociais, coordenado pelo professor Dr. Nei Alberto Salles Filho (UEPG/CNPq). Integrante do Comitê de implantação da Justiça Restaurativa no Município de Ponta Grossa, representando a OAB/PR. Advogada nas áreas cível e família, com experiência na aplicação das metodologias consensuais de resolução de conflito: Conciliação, Mediação e Justiça Restaurativa.

* Docente e pesquisador da Universidade Estadual de Ponta Grossa (UEPG/PR). Doutor em Educação pela Universidade Estadual de Ponta Grossa (UEPG/PR), com Pós-Doutorado em Ensino de Ciência e Tecnologia pela Universidade Tecnológica Federal do Paraná (UTFPR). Mestre em Educação pela Universidade Metodista de Piracicaba (UNIMEP/SP). Professor do Programa de Pós-Graduação em Ciências Sociais Aplicadas (M/D) da UEPG/PR. Professor do Programa de Pós-Graduação (M) em Educação Inclusiva (PROFEl-MEC/UEPG). Professor do Curso de Licenciatura em Educação Física (Graduação) da UEPG/PR. Pesquisador dos grupos:(1) Cultura de Paz, Direitos Humanos e Sustentabilidade e (2) Direitos Humanos, Cidadania e Políticas Públicas. Coordenador do Núcleo de Educação para a Paz (NEP/UEPG) que atua na formação de recursos humanos em alternativas às violências e qualificação das convivências em espaços educacionais e não-educacionais. Desenvolve estudos sobre Educação e Processos Sociais com ênfase nos temas: formação de professores; cultura de paz; educação para a paz; educação em direitos humanos; violências escolares; práticas restaurativas; conflitologia; ecoformação e sustentabilidade. Na dimensão teórica discute questões relacionadas à Teoria da Complexidade e as Epistemologias do Sul.

*** Possui graduação em Medicina pela Universidade Federal do Paraná, Residência Médica em Neurologia pelo Hospital de Clínicas/UFPR e mestrado em Ciências Biomédicas pela Universidade Estadual de Ponta Grossa. Atualmente é chefe do serviço e supervisor do Programa de Residência Médica em Neurologia do Hospital Universitário Regional dos Campos Gerais (HU-UEPG). Atua principalmente nas áreas de dor crônica, cefaleias e medicina do estilo de vida, coordena o serviço multiprofissional de dor crônica do HU-UEPG.
} 


\section{ABSTRACT}

This article presents the experience of the Center for Legal Practice at Centro Universitário Santa Amélia, in Ponta Grossa-PR, in the application of virtual peacebuilding circles in the context of social isolation caused by the COVID-19 pandemic. The circle is a method of Restorative Justice, which I define here as a simple but profound program for building more meaningful relationships and increasing emotional awareness. The negative psychological impacts of the coronavirus quarantine motivated the migration from face-to-face to online circles, understanding that circular practice can contribute to building a support network and strengthening bonds between people. With this objective, 96 circles were carried out in three semesters, involving 738 people, and the result was the perception that the circle, even virtually, is able to promote self-knowledge and interpersonal connection in a deep and meaningful way, contributing positively with mental health in the pandemic.

Keywords: Peace Building Circle; Holistic Vision; Normality.

\section{INTRODUÇÃO}

O contexto da pandemia da COVID-19 ${ }^{1}$ deixou ainda mais evidente a inter-relação entre todos os seres do planeta. Vive-se um vazio diante de tantas certezas científicas, narrativas, tecnologias e individualismos do ser humano. Um vírus tomou conta da agenda mundial e afetou todas as pessoas, países e ideologias.

Desde a confirmação da pandemia pela Organização Mundial da Saúde (OMS) em março de 2020, diversas medidas foram adotadas para evitar a contaminação pelo SARS-CoV-2. No Brasil, o Ministério da Saúde recomendou ações baseadas em medidas de higiene, uso de máscaras e isolamento social. A população foi surpreendida por medidas restritivas de mobilidade urbana, impondo a suspensão das aulas presenciais para instituições públicas e privadas; adoção de teletrabalho, fechamento do comércio e serviços não essenciais, redução na frota e horários de circulação do transporte público, entre outros. Tais medidas mudaram drasticamente o modo de viver das pessoas, repercutindo sobre a saúde mental da população.

A comunidade científica tem apresentado vários estudos que revelam os impactos psicológicos negativos da quarentena provocados pelo medo da infecção, a frustração e o tédio, a escassez de suprimentos e a carência de informações adequadas. Há previsão de que, a médio prazo, aumente a incidência de transtornos mentais como a ansiedade e a depressão, assim como doenças cardiovasculares. No entanto, há outro fator bastante preocupante que é o isolamento físico. Isto porque, o distanciamento recomendado agride diretamente o ser humano na sua necessidade de estar conectado com outras pessoas (BAUMEISTER; LEARY, 1995).

\footnotetext{
'Segundo a Folha Informativa da Organização Pan-americana da Saúde (OPAS): A COVID-19 é uma doença infecciosa causada pelo novo coronavírus (SARS-CoV-2) e tem como principais sintomas febre, cansaço e tosse seca. Alguns pacientes podem apresentar dores, congestão nasal, dor de cabeça, conjuntivite, dor de garganta, diarreia, perda de paladar ou olfato, erupção cutânea na pele ou descoloração dos dedos das mãos ou dos pés. Esses sintomas geralmente são leves e começam gradualmente. Algumas pessoas são infectadas, mas apresentam apenas sintomas muito leves. A maioria das pessoas (cerca de $80 \%$ ) se recupera da doença sem precisar de tratamento hospitalar. Uma em cada seis pessoas infectadas por COVID-19 fica gravemente doente e desenvolve dificuldade de respirar. As pessoas idosas e as que têm outras condições de saúde como pressão alta, problemas cardíacos e do pulmão, diabetes ou câncer, têm maior risco de ficarem gravemente doentes. No entanto, qualquer pessoa pode pegar a COVID-19 e ficar gravemente doente. Acessado em 26/06/2021 < https:// www.paho.org/pt/covid19>
} 
O pensamento dominante é de que o estresse é sempre ruim, porém, diante de uma pandemia global, evitá-lo completamente não é uma opção. Com uma mudança na mentalidade a na forma de avaliar a situação é possível melhorar o desempenho, promover enfrentamento ativo e otimizar a resposta ao estresse (CRUM; JAMIESON; AKINOLA, 2020; JAMIESON et al., 2018)and not surprisingly, the vast majority of interventions for coping with stress focus on reducing the frequency or severity of stressors. However, the effectiveness of stress attenuation is limited because it is often not possible to avoid stressors, and avoiding or minimizing stress can lead individuals to miss opportunities for performance and growth. Thus, during stressful situations, a more efficacious approach is to optimize stress responses (i.e., promote adaptive, approach-motivated responses.

Portanto, a saúde mental deve ser pensada a partir de abordagens holísticas, uma vez que, o ser humano é relacional e afeta e é afetado pelo contexto onde está inserido. Dentro desta perspectiva, a Justiça Restaurativa (JR) é uma epistemologia que valoriza práticas sociais que fortalecem os indivíduos e, estende-se às relações com os outros e com a comunidade. O círculo de construção de paz, como um dos métodos da JR, tem como pano de fundo a compreensão da interdependência entre os seres humanos e a sua necessidade de estar em bons relacionamentos. No contexto pandêmico, esse método é indicado para o fortalecimento de vínculos e a construção de redes de apoio.

Neste sentido, o Núcleo de Prática Jurídica (NPJ) do Centro Universitário Santa Amélia (UniSecal), na cidade de Ponta Grossa-PR, preocupado com os impactos psicológicos negativos da quarentena do coronavírus sobre a comunidade em geral, migrou os círculos presenciais para a modalidade on-line, entendendo que essa prática pode contribuir para a construção de rede de apoio e no fortalecimento de vínculo entre as pessoas, ainda que virtualmente. Com este objetivo os projetos do NPJ alcançaram, nos semestres de 2020.1, 2020.2 e 2021.1: professores universitários e estudantes da UniSecal; profissionais da saúde do Hospital Universitário Regional dos Campos Gerais e do Pronto Socorro Municipal; encarcerados da Cadeia Pública de Ponta Grosa e Penitenciária Estadual de Ponta Grossa e a comunidade.

A saúde mental e a JR têm uma estreita ligação nesse momento de pandemia. E foi a convergência desses pontos que levou à construção de ações concretas como os projetos do NPJ da UniSecal com os círculos virtuais de construção de paz.

\section{AMEAÇAS E OPORTUNIDADES PARA A SAÚDE MENTAL DURANTE A PANDEMIA DO COVID-19}

Com o início do isolamento físico ocorrido no Brasil a partir de março de 2020, as mudanças no estilo de vida são nítidas e possuem impactos atuais e futuros tanto na saúde física quanto mental (BROOKS et al., 2020; MATTIOLI; BALLERINI PUVIANI, 2020) O fechamento de espaços públicos e academias contribuiu para a redução da movimentação das pessoas e das atividades físicas em geral, apesar da orientação de exercícios em casa. Os hábitos alimentares foram modificados com o aumento do consumo de alimentos industrializados e refeições prontas e entregues em casa.

Um estudo preliminar analisou os impactos psicológicos negativos da quarentena do COVID-19 e destacou os sintomas de estresse pós-traumático, confusão e raiva (BROOKS et al., 2020) Os principais fatores estressores foram a duração do período de isolamento, o medo da infecção, a frustração e o tédio, a escassez de suprimentos e a carência de informações adequadas (BROOKS et al., 2020) Com isso, espera-se um aumento na incidência de transtornos mentais como a ansiedade e a 
depressão, assim como doenças cardiovasculares no período pós pandemia (MATTIOLI; BALLERINI PUVIANI, 2020).

O medo é uma reação emocional natural do ser humano e surge quando existe a percepção de uma ameaça, seja ela exterior ou proveniente dos seus pensamentos. Esta experiência pode gerar um aumento do autoritarismo e da intolerância à grupos externos ao seu grupo social (FELDMAN; STENNER, 1997; JACKSON et al., 2019) Tudo que vem de fora é visto como ruim ou prejudicial. Reduz a empatia com grupos sociais distantes (CIKARA; BRUNEAU; SAXE, 2011; HAN, 2018) e aumenta a desumanização (KTEILY; HODSON; BRUNEAU, 2016) Apesar de frequentemente considerado uma emoção negativa ele pode ser positivo, gerando mudança de comportamento quando o indivíduo percebe que é capaz de lidar com a ameaça (WITTE; ALLEN, 2000). Esforços coordenados entre indivíduos, comunidades e governos podem criar uma oportunidade para reduzir a discriminação, compartilhando valores e enviando uma mensagem de cooperação (BAVEL et al., 2020) Apesar do senso comum, as pessoas frequentemente tendem a cooperar mais do que competir frente à grandes desastres (DRURY, 2018) possivelmente relacionado ao fato das pessoas estarem compartilhando a mesma experiência (DRURY; COCKING; REICHER, 2009).

Em um ambiente competitivo, predomina o pensamento de soma zero, onde para alguém ganhar é necessário que outro perca. Porém, este pensamento não se enquadra durante uma pandemia, pois quando um indivíduo é infectado aumenta o risco de outras pessoas também serem infectadas. Ao acumular equipamentos de proteção individual acredita estar reduzindo a sua chance de se infectar, mas na verdade, ao deixar outras pessoas sem esse equipamento, acaba prejudicando a si mesmo. O ideal seria um pensamento de cooperação, favorecendo a proteção de todos e assim também a sua. (BAVEL et al., 2020)

Cooperação requer que as pessoas tolerem um certo custo individual no sentido de beneficiar outras pessoas, com isso existe um conflito entre a recompensa individual de curto prazo e a recompensa coletiva de longo prazo. Além disso, o coletivo pode representar a sua família, a comunidade local, nacional ou internacional, o que dificulta a tomada de decisão. As pesquisas sempre apontam que o indivíduo tende a priorizar a sociedade local à internacional, que está distante da sua realidade (MILINSKI et al., 2016; REINDERS FOLMER et al., 2012) As pessoas também são mais propensas a cooperar quando elas acreditam que os outros estão cooperando (FISCHBACHER; GÄCHTER; FEHR, 2001) ou percebem que estão sendo observadas (KRAFT-TODD et al., 2015).

A pandemia pode ser uma grande fonte de estresse, o cenário de incerteza em relação ao futuro pode gerar grande ansiedade relacionada à problemas econômicos e de saúde. O distanciamento físico e a grande quantidade de informações recebidas diariamente tendem a piorar a situação. $\mathrm{O}$ distanciamento recomendado agride diretamente o ser humano na sua necessidade de estar conectado com outras pessoas (BAUMEISTER; LEARY, 1995).

O pensamento dominante é de que o estresse é sempre ruim, porém, diante de uma pandemia global, evitá-lo completamente não é uma opção. Com uma mudança na mentalidade a na forma de avaliar a situação é possível melhorar o desempenho, promover enfrentamento ativo e otimizar a resposta ao estresse (CRUM; JAMIESON; AKINOLA, 2020; JAMIESON et al., 2018) Alguns trabalhos sugerem que essa mudança de mentalidade pode aumentar a possibilidade de "crescimento relacionado ao estresse", um fenômeno no qual experiências estressantes contribuem para reorganizar nossas prioridades, produzir relacionamentos mais profundos e uma maior apreciação pela vida (TEDESCHI; CALHOUN, 2004). 
Essa mudança de mentalidade individual está atrelada à ruptura de modelos científicos que silenciam sobre as conexões entre as coisas. O contexto pandêmico escancara a necessidade de um novo paradigma ${ }^{2}$ que emerge da vida, que parte de uma visão mais ampla que vai de uma visão de mundo mecanicista/reducionista para uma visão de mundo holística e ecológica. Neste processo, o mundo deixa de ser visto como uma máquina e passa ser compreendido como uma rede (CAPRA, 2014). Segundo CAPRA (2014), "Durante o século XX, a mudança do paradigma mecanicista para o paradigma ecológico se processou em diferentes formas e com diferentes velocidades em vários campos científicos", para o autor "A tensão básica é entre as partes e o todo. A ênfase nas partes tem sido chamada de mecanicista, reducionista ou atomística; a ênfase no todo, de holística, organísmica ou ecológica".

Portanto, a saúde mental deve ser pensada a partir de abordagens holísticas, uma vez que, o ser humano é relacional e afeta e é afetado pelo contexto onde está inserido. Dentro desta perspectiva, a JR é uma epistemologia que valoriza práticas sociais que fortalecem os indivíduos e, estende-se às relações com os outros e com a comunidade.

\section{JUSTIÇA RESTAURATIVA: UMA VISÃO DO BEM VIVER}

Os termos "justiça" e "restaurativa" não estão vinculados a pressupostos subjacentes das instituições sociais e políticas do ocidente. Esta prática social representa na sua essência a validação de saberes e valores característicos de muitos grupos indígenas, cuja compreensão política e social básica inclui o respeito no relacionamento entre indivíduo e comunidade e a conexão entre humanos e o mundo natural.

$\mathrm{A} \mathrm{JR}^{3}$ "contemporânea" representa o reconhecimento das tradições dos povos das primeiras nações e incorpora abordagens da comunicação não violenta, da escuta qualificada e da construção de consensos para atender as necessidades de indivíduos e da coletividade.

Dentro desta perspectiva holística, a descrição oferecida por Howard Zehr, como: "um tipo de sistema coerente de valores que nos dá uma visão do bem" parece ser um ponto de referência importante para garantir um tratamento saudável e bons resultados para os participantes (ELLIOTT, 2018).

Os valores humanos, que compõe este sistema ético a qual a JR encarna, são universais; daí a sua possível ligação com tantas tradições indígenas ou religiosas (ZEHR, 2018). Assim, há valores centrais que devem estar presentes em programas ou organizações de JR: respeito, honestidade e cuidado, são alguns deles (ELLIOTT, 2018).

A sociedade moderna tem, até certo ponto, subestimado a importância da conexão emocional através de relacionamentos estáveis em favor do uso de nossas habilidades mentais para aquisição de riqueza material e poder. Nós "praticamos" maneiras de estar em relacionamentos em nossas famílias, escolas, locais de trabalho e comunidades que são profundamente insatisfatórias e que tornam mais difícil nos conectarmos um com o outro e com nosso eu verdadeiro (BOYES-WATSON; PRANIS, 2011).

\footnotetext{
${ }^{2}$ Segundo a definição de Thomas Khun, o paradigma científico é como uma constelação de realizações - conceitos, valores, técnicas etc. - compartilhadas por uma comunidade científica e usadas por essa comunidade para definir problemas e soluções legítimos (CAPRA, 2014).

${ }^{3}$ A justiça restaurativa como metodologia é uma inovação teórica que data da década de 70, na América Anglo Saxônica, a qual resgata saberes da ancestralidade humana que utilizavam o diálogo como principal instrumento para organização da convivência coletiva. Esses conhecimentos foram denominados "Justiça Restaurativa" e foram cunhados pela primeira vez por Albert Eglash no Canadá no encontro entre vítima e ofensor em um caso de crime violento (ELLIOT, 2018, p.108).
} 
Na medida em que esses conhecimentos ficaram à margem da ciência moderna e daquilo que era conhecido como "verdade" pelo mundo, expôs-se a própria contradição do discurso dominante, pois se conhecimento significa "a capacidade de as pessoas se apropriarem do mundo em geral", boa parte do mundo não se reconheciam neste modelo e assim não o legitimavam (SANTOS; MENESES; PEIXOTO, 2018).

A ciência então ficou distante da realidade e da sociedade, que continuou a se valer do conhecimento da prática cotidiana da vida. Essa visão de mundo, que descola o conhecimento científico da realidade da sociedade, há algum tempo, não consegue dar respostas às necessidades humanas individuais e coletivas. Afinal, segundo Boaventura (2018, p.32) "O sentido da vida não faz parte da ciência, os objetivos finais da vida não fazem parte da ciência; a eficácia da ciência advém-lhe da forma como reduz os problemas relevantes àqueles que podem ser resolvidos cientificamente".

Na busca por respostas que a ciência moderna tem dificuldade de incorporar, profissionais de várias áreas têm explorado práticas alternativas, como a JR, nas suas vertentes: conferência de grupo familiar, encontro vítima-ofensor e círculo de construção de paz, cujo foco está nos relacionamentos, na consciência emocional e na competência emocional.

No Brasil a JR foi regulamentada pela resolução n²25/2016 do Conselho Nacional de Justiça como uma política pública do Poder Judiciário. No âmbito judicial a JR é um método consensual de resolução de conflito e dentre suas vertentes acima citadas, a mais utilizada é o círculo de construção de paz por influência da norte-americana Kay Pranis, responsável pelas primeiras capacitações no Brasil. No entanto, os círculos não são aplicados somente para situações de conflitos, há outras modalidades, como os círculos de diálogo, com foco no fortalecimento de vínculos e construção de redes de apoio. Esse último foi o modelo mais utilizado nos projetos do NPJ por conta das necessidades emocionais que surgiram a partir do afastamento social provocado pela quarentena.

O contexto da pandemia da COVID-19 deixou ainda mais evidente essa inter-relação entre todos os seres do planeta. Vive-se um vazio diante de tantas certezas científicas, narrativas, tecnologias e individualismos do ser humano. Um vírus tomou conta da agenda mundial e afetou todas as pessoas, países e ideologias. O coronavírus, junto com a angústia e a tristeza das mortes, internações e medos, trouxe, ao mesmo tempo, a necessidade de revisitar/rever muitas coisas daquilo que chamamos de "normalidade".

Essa suposta "normalidade" estaria no estilo de vida da maioria das pessoas da comunidade planetária. Mas, de que estilo de vida falamos? Da alimentação predominantemente industrializada? Do sedentarismo? Das noites mal dormidas? Do uso exagerado de substâncias químicas? Das crises de ansiedade devido ao estresse? Dos relacionamentos superficiais e individualistas? Essa "normalidade" trouxe a humanidade até aqui.

A resposta parece clara, separados quase todos perdem e juntos a maioria pode superar! Edgar Morin, filósofo contemporâneo, neste momento em quarentena na França - sabiamente disse: "Não há dúvida que precisamos de racionalidade em nossas vidas. Mas temos a necessidade de afetividade, ou seja, de laços, de plenitude, de alegria, de amor, de exaltação, de jogo, de eu, de nós" (MORIN, 2015).

As palavras de Edgar Morin dão pistas de como começar a digerir este momento que trará mudanças profundas ao nosso sentido de "normalidade". O primeiro ponto é deixar a incerteza chegar

\footnotetext{
${ }^{4}$ Vale pensar no conceito de "normose" como a "patologia da normalidade", analisado por Pierre Weil, Jean Yves Leloup e Roberto Crema em livro do mesmo nome (2014).
} 
e acolher o momento que não tem prazo determinado para acabar. O foco é no tempo presente, da preservação da vida, da saúde física, da saúde mental, das relações humanas e especialmente da empatia e da solidariedade com pessoas e grupos que estão vulneráveis mental e materialmente.

Portanto, conforme argumentamos até aqui, a saúde mental e a JR têm uma estreita ligação nesse momento de pandemia. Os estudos expostos no primeiro tópico reforçam que saúde não é só cuidar de doença, mas é resultado de escolhas que promovam e restabeleçam o equilíbrio físico e mental, a JR apresenta que o bem viver individual está atrelado ao contexto coletivo. Assim, o humano, como ser social, só encontra sentido na vida na relação com o outro. Estes são os pontos de ligação que nos levaram à construção de ações concretas como o projeto com os círculos de construção de paz.

\section{CÍRCULO DE CONSTRUÇÃO DE PAZ: REFLEXŌES SOBRE A NORMALIDADE}

Como um dos métodos da JR, o círculo de construção de paz oferece um programa simples, mas profundo, para construir relacionamentos mais significativos e ampliar a consciência emocional.

Organizados em formato circular, as pessoas são convidadas a compartilhar sentimentos e pensamentos. Cada participante contribui na sua medida e no seu tempo, enquanto os outros oferecem a escuta ativa. A prática circular encoraja a atenção plena, pois as pessoas focam naquilo que os outros estão dizendo, sem fazer várias outras coisas ao mesmo tempo, e sem interromper. O ritmo é muito mais lento do que a maioria das formas de comunicação, concedendo tempo para pensar e para dizer o que se possa querer dizer de forma plena (BOYES-WATSON; PRANIS, 2011).

O diálogo coletivo no espaço circular simboliza os princípios fundamentais da liderança compartilhada: igualdade, conexão e inclusão, e proporciona foco, comprometimento e participação de todos em um ambiente seguro e respeitoso. Esse espaço favorável permite aos participantes uma fala espontânea e sincera que promove a conexão e a empatia. Esse modo de ver a vida parte do pressuposto de que existe um desejo humano universal de estar ligado aos outros de forma positiva (PRANIS, 2010).

De forma prática, o círculo pode ajudar a fortalecer a família, dando a seus membros a oportunidade de reconhecer suas próprias potencialidades e recursos. Pode ajudar a redirecionar a cultura de jovens para uma direção mais positiva, permitindo que sejam fonte de apoio e sabedoria uns para os outros. Pode contribuir para o fortalecimento de vínculos entre colegas de trabalho, que reconhecem os seus dons complementares. Enfim, o círculo de construção de paz é um lugar para se adquirir habilidades e hábitos para formar relacionamentos saudáveis, não só dentro do círculo, mas também fora dele (BOYES-WATSON; PRANIS, 2011).

O fio condutor da JR está no sistema de valores que nos conduzem a repensar as escolhas "normais" que nos conduziram à desconexão do 'eu' e do 'nós', ao empobrecimento social, à degradação ambiental, à solidão, à desesperança e a desistir de sentir paz. Neste contexto, o NPJ da UniSecal, na cidade de Ponta Grossa-PR, vocacionado à aplicação dos métodos consensuais de solução de conflitos, desde o ano de 2017, firmou parcerias com instituições públicas e privadas para desenvolver projetos relacionados à prática circular. No entanto, a partir do mês março de 2019, com o início da pandemia, os círculos migraram para o formato virtual.

Segue tabela descritiva com os projetos, participantes e período da aplicação dos círculos virtuais no período da pandemia. 
Tabela 1: Círculos virtuais realizados pelo NPJ da UniSecal nos semestres:

2020.1, 2020.2 e 2021.1.

\begin{tabular}{|c|c|c|c|c|}
\hline PROJETOS DE EXTENSĀO & INSTITUIÇÃO PARCEIRA & PERFIL/ N DE PARTICIPANTES & N DE CÍRCULOS & ANO/SEMESTRE \\
\hline JR Institucional & UniSecal & $\begin{array}{l}\text { Professores do Curso de Direito (8) } \\
\text { Facilitadores do NPJ (4) } \\
\text { Representantes dos alunos do } \\
\text { Curso de Direito (9) } \\
\text { Alunos do } 10^{\circ} \text { período do Curso } \\
\text { de Direito (56) }\end{array}$ & 9 & \multirow[t]{6}{*}{2020.1} \\
\hline $\begin{array}{l}\text { Círculo de Fortalecimento } \\
\text { de Vínculo }\end{array}$ & $\begin{array}{l}\text { Hospital Universitário } \\
\text { Regional dos Campos } \\
\text { Gerais (HU) }\end{array}$ & $\begin{array}{l}\text { Residentes de Fisioterapia (13) } \\
\text { Residentes da Reabilitação (3) } \\
\text { Coordenação da Residência } \\
\text { Multidisciplinar (6) } \\
\text { Equipe multiprofissional de } \\
\text { atendimento ao COVID (5) }\end{array}$ & 5 & \\
\hline $\begin{array}{l}\text { Círculo de Construção de } \\
\text { Rede de Apoio }\end{array}$ & $\begin{array}{l}\text { Pronto Socorro Municipal - } \\
\text { Hospital Municipal Doutor } \\
\text { Amadeu Puppi }\end{array}$ & Fisioterapeutas (9) & 1 & \\
\hline $\begin{array}{l}\text { Círculo Virtual (testes do } \\
\text { modelo virtual em parceria } \\
\text { com o CEJUSC-PG) }\end{array}$ & $\begin{array}{l}\text { Centro Judiciário de } \\
\text { Solução de Conflitos e } \\
\text { Cidadania de Ponta } \\
\text { Grossa (CEJUSC-PG) }\end{array}$ & Facilitadores (19) & 3 & \\
\hline $\begin{array}{l}\text { Círculo de Construção de } \\
\text { Rede de Apoio }\end{array}$ & Município de Ipiranga-PR & Facilitadores (3) & 1 & \\
\hline Roda de Prosa & Comunidade & Comunidade (5) & 1 & \\
\hline JR Institucional & UniSecal & $\begin{array}{l}\text { Alunos do } 10^{\circ} \text { período do Curso } \\
\text { de Direito }(56)\end{array}$ & 8 & \multirow[t]{6}{*}{2020.2} \\
\hline Projeto Expressão & $\begin{array}{l}\text { Cadeia Pública de Ponta } \\
\text { Grossa Hildebrando de } \\
\text { Souza }\end{array}$ & Presos/Presas (78) & 16 & \\
\hline $\begin{array}{l}\text { Projeto Travessia (projeto } \\
\text { com } 4 \text { encontros com } 12 \\
\text { participantes por vez) }\end{array}$ & $\begin{array}{l}\text { Penitenciária Estadual de } \\
\text { Ponta Grosa }\end{array}$ & Presos (24) & 8 & \\
\hline $\begin{array}{l}\text { Círculo de Conflito } \\
\text { (parceria em dois processos } \\
\text { com o CEJUSC-PG) }\end{array}$ & CEJUSC-PG & Comunidade (7) & 2 & \\
\hline $\begin{array}{l}\text { Círculo Bem-Estar na Fibro } \\
\text { (projeto com } 10 \text { encontros } \\
\text { com temas diferentes) }\end{array}$ & $\begin{array}{l}\text { Hospital Universitário } \\
\text { Regional dos Campos } \\
\text { Gerais (HU) }\end{array}$ & $\begin{array}{l}\text { Pacientes (7) } \\
\text { Profissionais da saúde (3) }\end{array}$ & 10 & \\
\hline Roda de Prosa & Comunidade & Comunidade (1) & 1 & \\
\hline JR Institucional & UniSecal & $\begin{array}{l}\text { Alunos do } 1^{\circ} \text { período (49) } \\
\text { Alunos do } 10^{\circ} \text { período (46) } \\
\text { Círculo de Conflito alunos do } 7^{\circ}(8) \\
\text { Círculo Good Vibes com } \\
\text { professores do Curso de Direito (22) }\end{array}$ & 11 & \multirow[t]{5}{*}{2021.1} \\
\hline $\begin{array}{l}\text { Círculo de Fortalecimento } \\
\text { de Vínculo }\end{array}$ & $\begin{array}{l}\text { Hospital Universitário } \\
\text { Regional dos Campos } \\
\text { Gerais (HU) }\end{array}$ & $\begin{array}{l}\text { Residentes da reabilitação (11) } \\
\text { Residentes da residência } \\
\text { multidisciplinar (28) }\end{array}$ & 5 & \\
\hline Círculo de Auto-Cuidado & Comunidade & Comunidade (12) & 2 & \\
\hline Círculo Kintsug & Comunidade & Comunidade (9) & 2 & \\
\hline Projeto Expressão & $\begin{array}{l}\text { Cadeia Pública de Ponta } \\
\text { Grossa Hildebrando de } \\
\text { Souza }\end{array}$ & Presas (51) & 11 & \\
\hline
\end{tabular}

Fonte: Dados obtidos pelos autores dos relatórios dos Projetos de Extensão do NPJ da UniSecal.

As inseguranças física e emocional provocadas pela pandemia impuseram um ritmo acelerado às pessoas na intenção de tentar deixar tudo "normal": calendários, compromissos, dietas, atividade física, cursos on-line, que, obviamente, são importantes. Mas, para qual "normalidade"? Àquela em 
que a jornada solitária não nos conecta enquanto seres vivos e dependentes uns dos outros? $\mathrm{O}$ vácuo pandêmico nos empurra a buscar novas referências/modelos de ver a vida. Que vida pessoal pretendemos cultivar com as pessoas da família? E com os colegas de trabalho? Como devemos usar nossa formação profissional para contribuir com um mundo melhor, mais humano, menos desigual e sem tantas injustiças?

Neste novo contexto, o círculo de construção de paz é uma tecnologia social adequada para ajudar as pessoas a tomarem decisões melhores a respeito do seu futuro. Isso porque, é espaço próprio para refletir sobre novos modelos/alternativa para o bem viver individual e coletivo.

Como oportunidade de ressignificar o próprio método, que tradicionalmente considera a presença física como um dos elementos de conexão emocional entre os participantes, o círculo foi restruturado para ser aplicado de forma virtual pela internet, pelo aplicativo $\mathrm{ZOOM}^{\circledR}$ ou Google Meet. A mescla entre os métodos e a forma de aplicá-los não é uma novidade na JR. Cada cultura demanda adaptações às suas realidades. O que não se pode perder de vista são os valores subjacentes. $\mathrm{O}$ argumento é simples: a prática restaurativa está embasada em valores de respeito, honestidade e cuidado, e se as e pessoas que foram atendidas pelo processo relatam que foram tratadas com respeito, honestidade e carinho, então o objetivo foi alcançado (ELLIOTT, 2018).

Os círculos de construção de paz desenvolvidos pelo NPJ foram então repensados para atender essas novas/velhas demandas provocadas pelo isolamento físico da pandemia. Ainda que de forma virtual e sem a presença física, o círculo manteve a mesma estrutura didática, nutrindo os mesmos valores de conexão, empatia e atenção plena.

No entanto, algumas questões práticas foram alteradas: a mais significativa foi o uso coletivo da sala virtual do aplicativo $\mathrm{ZOOM}^{\circledR}$ ou Google Meet. As pessoas são convidadas para ingressar em hora e dia previamente combinados. As instruções para o círculo virtual incluem a escolha de um lugar silencioso para acessar a plataforma, tendo em vista que a atividade exige tranquilidade e plena atenção; que os vídeos devam permanecer ligados para haver o contato visual; que a fala é regulada pela ordem alfabética dos nomes, assim, o microfone só poderá ser ativado quando for a vez de falar; que ninguém está autorizado a gravar o encontro, tendo em vista que tudo o que é compartilhado é confidencial.

O NPJ iniciou os atendimentos neste novo formato, a princípio, para atender demandas de alunos e profissionais da faculdade relacionadas à saúde mental. No dia 15 de abril de 2020 foi realizado o primeiro círculo virtual com os professores do Curso de Direito. A partir de então os projetos já existentes (JR Institucional, Círculo de Fortalecimento de Vínculos, Projeto Travessia e Projeto Expressão) e os novos projetos (Círculo de Construção de Rede de Apoio, Roda de Prosa, Círculo de Conflitos, Círculo de Auto-Cuidado, Círculo Kintsug e Círculo de Bem-Estar na Fibro) foram todos realizados no formato virtual.

Após três semestres (2020.1, 2020.2 e 2021.1), foram realizados pelo NPJ da UniSecal 96 círculos, envolvendo 738 pessoas. Os projetos alcançaram: professores universitários e estudantes da UniSecal; profissionais da saúde do Hospital Universitário Regional dos Campos Gerais e do Pronto Socorro Municipal; encarcerados da Cadeia Pública de Ponta Grosa e Penitenciária Estadual de Ponta Grossa e a comunidade.

Em média cada círculo tinha de 5 a 12 pessoas e a duração era em torno de uma hora e meia a duas horas. Todos os participantes eram convidados previamente para participar da prática circular. A 
grande parte das pessoas que participaram do círculo nunca havia vivenciado essa experiência antes. Em um primeiro momento, o estranhamento com o manuseio da tecnologia (celular ou computador) e com a plataforma digital (ZOOM ${ }^{\circledR}$ ou Google Meet) deixavam os participantes mais tensos e ansiosos, mas à medida que se habituavam e se sentiam mais seguros, o diálogo, incialmente, mais superficial, desdobrava-se e se aprofundava.

No ambiente hospitalar o feedback dos coordenadores foi positivo. Foi relatado melhora no relacionamento entre os residentes com a diminuição dos conflitos na rotina da enfermaria, redução nas ausências e um maior comprometimento com as atividades da residência. Já os participantes relataram que as histórias compartilhadas no círculo, com escuta qualificada e sem pressa, permitiram que os relacionamentos a partir desse encontro fossem mais sinceros, empáticos e acolhedores. $\mathrm{Na}$ universidade, a percepção de alunos e professores foi o sentimento de acolhimento proporcionado no círculo e a satisfação de conhecer os colegas com mais calma e profundidade. Os conflitos entre alunos e professores resolvidos no círculo não tiveram outros desdobramentos, sugerindo que os envolvidos tiveram suas necessidades satisfatoriamente atendidas. Os círculos aplicados com a comunidade e com as pessoas encarceradas também foram satisfatórios para os participantes, ao final, no check-out, quando é perguntado como foi a experiência com a prática circular, as pessoas responderam dizendo que estavam melhores de quando chegaram.

Como resultado desses três semestres de aplicação dos círculos virtuais algumas dificuldades podem ser apontadas: a instabilidade da internet dos participantes, o manuseio das plataformas digitais pelos participantes, a garantia da atenção plena dos participantes e a garantia que o participante está isolado de outras pessoas por conta do sigilo. Também podem ser indicados alguns cuidados para evitar algumas dessas dificuldades: no caso de círculo de conflito, realização de pré-encontros individuais para melhor explicação da ferramenta virtual e da importância da atenção plena e do sigilo do diálogo; nos círculos de relacionamento, enviar aos participantes orientações do uso da plataforma virtual e das diretrizes comportamentais sobre a plena atenção e o sigilo do diálogo.

Ainda assim, os facilitadores do $\mathrm{NPJ}^{5}$ sinalizaram que a essência do círculo presencial foi mantida. A interconexão gerada nos encontros esteve presente, assim como a empatia e o valor de cuidado um para com o outro. Os relatos da autorreflexão gerados nos encontros confirmam e reconhecem a interdependência mútua na batalha para o bem viver. Ficou evidente que independente da idade, lugar, gênero, condição social, posição hierárquica, é possível juntar pessoas como iguais para falar de assuntos difíceis em uma atmosfera e preocupação com todos.

Ao final dos círculos virtuais, os participantes apontaram novas referências/habilidades e quem sabe novas "normalidades" aprendidas: a escuta empática, o não julgamento, falar sem pressa e sem interromper, a atenção plena e fala sincera. Essa experiência ensina que o isolamento físico não significa o isolamento social, e que o uso alternativo das ferramentas virtuais pode ajudar a fortalecer e criar relacionamentos com um senso de conexão tão forte quanto aqueles fisicamente construídos, porque a qualidade dessas interações é o que realmente importa.

\footnotetext{
${ }^{5}$ Os facilitadores são professores, alunos, funcionários da UniSecal e pessoas da comunidade que fizeram o curso de capacitação no método do círculo de construção de paz da justiça restaurativa, conforme preconiza a Resolução n²25/2016 do Conselho Nacional de Justiça.
} 


\section{CONSIDERAÇÕES}

A pandemia do COVID-19 é um fenômeno social global cujas repercussões e desdobramentos só serão conhecidos ao longo dos anos. Mesmo após um ano e meio da sua confirmação pela OMS (20 de março de 2019), ainda há muitas dúvidas na contenção da doença. Segundo o site oficial da $\mathrm{OMS}^{6}$, no dia 30 de junho de 2021, há no mundo 181.521.067 casos confirmados, incluindo 3.937.437 de mortes. No Brasil são 18.448.402 casos confirmados e 514.092 mortes. A situação é extremamente preocupante na medida em somente $12,7 \%$ da população brasileira foi vacinada com as doses ${ }^{7}$ necessárias para a imunização.

Tornou-se rotina para a população mundial conviver com o sofrimento provocado pelas mortes, o medo da contaminação, a insegurança das informações e, principalmente, o isolamento social, confirmando as pesquisas sobre o impactado negativo na saúde física e mental da maioria das pessoas.

Tem-se compreendido também, que o modelo tradicional de compreender os fenômenos sociais precisam considerar o contexto e sua rede de aspectos que interferem no jogo da vida. Assim, a saúde mental na pandemia deve ser analisada sob uma visão mais ampla e multidisciplinar, que conduza a humanidade a repensar as escolhas "normais" que nos conduziram à desconexão do 'eu' e do 'nós', ao empobrecimento social, à degradação ambiental, à solidão, à desesperança e a desistir de sentir paz.

A JR é um caminho epistemológico alternativo na medida em que considera como válido as experiências que valorizam as conexões emocionais na forma como se configuram os relacionamentos humanos e desses com todo a forma de vida no planeta. Dentre os métodos da JR, os círculos de construção de paz resgatam valores da ancestralidade para reforçar o que é intrínseco a todo ser humano: o desejo universal de estar ligado aos outros de forma positiva (PRANIS, 2010).

No contexto do isolamento pandêmico, as práticas circulares no formato virtual tornaram-se uma estratégia para que as pessoas pudessem resgatar o contato emocional na relação com o outro, ajudando no enfrentamento ativo e otimizar a resposta ao estresse vivido neste momento.

A abordagem dos círculos virtuais aplicados a profissionais e pessoas da comunidade pelos projetos do NPJ da UniSecal, inicialmente, foi um grande desafio devido aos receios sobre como garantir que princípios, como do sigilo, fosse de fato respeitado. Havia também a insegurança com o manuseio das ferramentas tecnológicas e a instabilidade da internet. A final, as pessoas conseguiriam estar seguras o suficiente para se conectarem umas com as outras?

Após três semestres de acompanhamento e aplicação dos círculos virtuais, a conclusão dos facilitadores foi de que o círculo, mesmo virtual, é uma excelente estratégia para construção de espaços seguros para os participantes compartilharem suas estórias e sentimentos, ajudando no autoconhecimento e na conexão interpessoal de uma forma profunda e significativa, contribuindo positivamente com a saúde mental das pessoas na pandemia.

\footnotetext{
${ }^{6}$ Acesso em 30/06/2021 < https://covid19.who.int/.

7 Informação do site do Ministério da Saúde acessado em 30/06/2021 < https://qsprod.saude.gov.br/extensions/DEMAS_ C19Vacina/DEMAS_C19Vacina.html.
} 


\section{REFERÊNCIAS}

BAUMEISTER, R. F.; LEARY, M. R. The need to belong: Desire for interpersonal attachments as a fundamental human motivation. Psychological Bulletin, [S. I.], v. 117, n. 3, p. 497-529, 1995. Disponível em: https://doi.org/10.1037/0033-2909.117.3.497

BAVEL, J. J. Van et al. Using social and behavioural science to support COVID-19 pandemic response. Nature Human Behaviour, [S. I.] , p. 1-12, 2020. Disponível em: https://doi.org/10.1038/ s41562-020-0884-Z

BOYES-WATSON, C.; PRANIS, K. No Coração da Esperança: Guia de Práticas Circulares. Porto ALegre: Tribunal de Justiça do Estado do Rio Grande do Sul, 2011 . E-book.

BROOKS, S. K. et al. The psychological impact of quarantine and how to reduce it: rapid review of the evidence. The Lancet, [S. I.], v. 395, n. 10227, p. 912-920, 2020. Disponível em: https://doi. org/10.1016/S0140-6736(20)30460-8

CIKARA, M.; BRUNEAU, E. G.; SAXE, R. R. Us and Them: Intergroup Failures of Empathy. Current Directions in Psychological Science, [S. I.] , v. 20, n. 3, p. 149-153, 2011 . Disponível em: https://doi. org/10.1177/0963721411408713. Acesso em: 24 maio. 2020.

CRUM, A. J.; JAMIESON, J. P.; AKINOLA, M. Optimizing stress: An integrated intervention for regulating stress responses. Emotion (Washington, D.C.), [S. I.], v. 20, n. 1, P. 120-125, 2020. Disponível em: https://doi.org/10.1037/emo0000670

DING, Y.; DAI, J. Advance in Stress for Depressive Disorder. In: Advances in Experimental Medicine and Biology. [S. I.]: Springer, 2019. v. 1180p. 147-178. E-book. Disponível em: https://doi. org/10.1007/978-981-32-9271-0_8. Acesso em: 23 maio. 2020.

DRURY, J. The role of social identity processes in mass emergency behaviour: An integrative review. European Review of Social Psychology, [S. I.] , v. 29, n. 1, p. 38-81, 2018. Disponível em: https://doi.or $\mathrm{g} / 10.1080 / 10463283.2018 .1471948$

DRURY, J.; COCKING, C.; REICHER, S. The Nature of Collective Resilience: Survivor Reactions to the 2005 London Bombings. International Journal of Mass Emergencies and Disasters, [S. I.], v. 27, n. 1, p. 66-95, 2009.

ELLIOTT, E. M. Segurança e Cuidado: Justiça Restaurativa e sociedades saudáveis. 1 ed. ed. São Paulo: Palas Athena, 2018. E-book.

FELDMAN, S.; STENNER, K. Perceived Threat and Authoritarianism. Political Psychology, [S. I.], v. 18, n. 4, p. 741-770, 1997. Disponível em: https://doi.org/10.1111/0162-895X.00077. Acesso em: 24 maio. 2020.

FISCHBACHER, U.; GÄCHTER, S.; FEHR, E. Are people conditionally cooperative? Evidence from a public goods experiment. Economics Letters, [S. I.] , v. 71, n. 3, p. 397-404, 2001. Disponível em: https://doi.org/10.1016/S0165-1765(01)00394-9

HAN, S. Neurocognitive Basis of Racial Ingroup Bias in Empathy. [S. I.]: Elsevier Ltd, 2018. Disponível em: https://doi.org/10.1016/j.tics.2018.02.013

HAWKLEY, L. C.; CACIOPPO, J. T. Loneliness matters: A theoretical and empirical review of consequences and mechanisms. Annals of Behavioral Medicine, [S. I.], v. 40, n. 2, p. 218-227, 2010. Disponível em: https://doi.org/10.1007/s12160-010-9210-8 
JACKSON, J. C. et al. Ecological and cultural factors underlying the global distribution of prejudice. PLOS ONE, [S. I.], v. 14, n. 9, p. e0221953, 2019. Disponível em: https://doi.org/10.1371/journal. pone.0221953. Acesso em: 24 maio. 2020.

JAMIESON, J. P. et al. Optimizing stress responses with reappraisal and mindset interventions: an integrated model. [S. I.]: Routledge, 2018. Disponível em: https://doi.org/10.1080/10615806.2018.1442615

JETTEN, J. et al. Advancing the social identity approach to health and well-being: Progressing the social cure research agenda. European Journal of Social Psychology, [S. I.], v. 47, n. 7, p. 789-802, 2017. Disponível em: https://doi.org/10.1002/ejsp.2333. Acesso em: 23 maio. 2020.

KELLY, J.; KARLSEN, M. C.; LIANOV, L. Establishing Competencies for Physicians Who Specialize in the Practice of Lifestyle Medicine. American Journal of Lifestyle Medicine, [S. I.] , v. 14, n. 2, p. 150-154, 2020. Disponível em: https://doi.org/10.1177/1559827619856542

KRAFT-TODD, G. et al. Promoting cooperation in the field. [S. I.]: Elsevier Ltd, 2015. Disponível em: https://doi.org/10.1016/j.cobeha.2015.02.006

KTEILY, N.; HODSON, G.; BRUNEAU, E. They see us as less than human: Metadehumanization predicts intergroup conflict via reciprocal dehumanization. Journal of Personality and Social Psychology, [S. I.] , v. 110, n. 3, p. 343-370, 2016. Disponível em: https://doi.org/10.1037/pspa0000044

KUSHNER, R. F.; SORENSEN, K. W. Lifestyle medicine: The future of chronic disease management. [S. I.]: Curr Opin Endocrinol Diabetes Obes, 2013. Disponível em: https://doi.org/10.1097/01. med.0000433056.76699.5d

KVAM, S. et al. Exercise as a treatment for depression: A meta-analysis. [S. I.]: Elsevier B.V., 2016. Disponível em: https://doi.org/10.1016/j.jad.2016.03.063

LUO, Y. et al. Loneliness, health, and mortality in old age: A national longitudinal study. Social Science and Medicine, [S. I.], v. 74, n. 6, p. 907-914, 2012. Disponível em: https://doi.org/10.1016/j. socscimed.2011.11.028

MATTIOLI, A. V.; BALLERINI PUVIANI, M. Lifestyle at Time of COVID-19: How Could Quarantine Affect Cardiovascular Risk. American Journal of Lifestyle Medicine, [S. I.] , p. 240-242, 2020. Disponível em: https://doi.org/10.1177/1559827620918808

MCEWEN, B. S.; KARATSOREOS, I. N. Sleep deprivation and circadian disruption: Stress, allostasis, and allostatic load. [S. I.]: W.B. Saunders, 2015. Disponível em: https://doi.org/10.1016/j. jsmc.2014.11.007

Medicina do Estilo de Vida | CBMEV - Colégio Brasileiro de Medicina do Estilo de Vida. [s. I.], [s. d.]. Disponível em: https://www.cbmev.org.br. Acesso em: 23 maio. 2020.

MILINSKI, M. et al. Humans choose representatives who enforce cooperation in social dilemmas through extortion. Nature Communications, [S. I.], v. 7, n. 1, p. 1-9, 2016. Disponível em: https://doi. org/10.1038/ncomms 10915

MORIN, E. Ensinar a viver. [S. I.]: Editora Sulina, 2015. E-book.

ORGANIZATION, W. H. Global recommendations on physical activity for health. [S. I.]: World Health Organization, 2010. E-book.

PRANIS, K. Processos Circulares. 1 ed. ed. São Paulo: Palas Athena, 2010. E-book. 
REINDERS FOLMER, C. P. et al. One for all: What representing a group may do to us. Journal of Experimental Social Psychology, [S. I.] , v. 48, n. 5, p. 1047-1056, 2012. Disponível em: https://doi. org/10.1016/j.jesp.2012.04.009. Acesso em: 24 maio. 2020.

SAGNER, M. et al. Lifestyle medicine potential for reversing a world of chronic disease epidemics: From cell to community. International Journal of Clinical Practice, [S. I.], v. 68, n. 11, p. 1289-1292, 2014. Disponível em: https://doi.org/10.1111/ijcp.12509

SANTOS, B. de S.; MENESES, M. P.; PEIXOTO, C. Na oficina do sociólogo artesão : aulas 2011-2016. 1 ed. ed. São Paulo: Cortez, 2018. E-book.

TEDESCHI, R. G.; CALHOUN, L. G. Posttraumatic Growth: Conceptual Foundations and Empirical Evidence. [S. I.]: Routledge, 2004. Disponível em: https://doi.org/10.1207/s15327965pli1501_01

WEIL, P.; LELOUP, J.-Y.; CREMA, R. Normose: A Patologia da Normalidade. [S. I.: s. n.]. E-book. WILLIAMS, W. C. et al. Interpersonal emotion regulation: Implications for affiliation, perceived support, relationships, and well-being. Journal of Personality and Social Psychology, [S. I.], v. $115, \mathrm{n}$. 2, p. 224-254, 2018. Disponível em: https://doi.org/10.1037/pspi0000132

WITTE, K.; ALLEN, M. A meta-analysis of fear appeals: Implications for effective public health campaigns. Health Education and Behavior, [S. I.], v. 27, n. 5, p. 591-615, 2000. Disponível em: https://doi.org/10.1177/109019810002700506

YARIBEYGI, H. et al. The impact of stress on body function: A review. [S. I.]: Leibniz Research Centre for Working Environment and Human Factors, 2017. Disponível em: https://doi.org/10.17179/ excli2017-480

YEH, B.-I.; KONG, I. D. The Advent of Lifestyle Medicine. Journal of lifestyle medicine, [S. I.], v. 3, n. 1, p. 1-8, 2013.

ZEHR, H. Trocando as lentes : justiça restaurativa para o nosso tempo. 3 ed. ed. São Paulo: Palas Athena, 2018. E-book. 